\title{
Um Modelo de Autômatos Celulares para o Espalhamento Geográfico de Epidemias
}

\author{
L.R. EMMENDORFER ${ }^{1}$, L.A.D. RODRIGUES ${ }^{2}$, UNIJUÍ, Programa de Pós-
} Graduação em Modelagem Matemática, Cx.P. 560, 98700-000 Ijuí, RS, Brasil.

Resumo. A teoria clássica de Epidemiologia, em geral, não considera a dimensão espacial de transmissão da doença e em vez disso, assume que todo indivíduo tem a mesma probabilidade de encontrar os outros. Modelos espaciais são indispensáveis para analisar questões tais como a velocidade com a qual uma doença se espalha e o padrão espacial de prevalência da mesma. Holmes [3] considera um modelo de autômatos celulares (AC) para o espalhamento geográfico de epidemias onde a transmissão é estritamente local, e compara este modelo com um outro onde a transmissão é global. Em nosso trabalho estudamos outras formas de representar a transmissão da doença entre os indivíduos em um modelo AC. Além da vizinhança "local", que inclui os indivíduos geometricamente mais próximos, incluímos efeitos "não-locais" que representam os contatos aleatórios que podem ocorrer entre indivíduos distantes. A variação relativa entre as escalas de transmissão local e global permite analisar diversas situações de interesse na dinâmica de epidemias.

\section{Introdução}

A representação de epidemias através de modelos matemáticos têm sido feita há quase um século. Dentre os modelos existentes para representar a dinâmica de epidemias devemos destacar o modelo compartimental de Kermack e McKendrick [4]:

$$
\begin{aligned}
\frac{d S}{d t} & =-\beta S I-b S I+b, \\
\frac{d I}{d t} & =\beta S I-\mu I-b I, \\
\frac{d R}{d t} & =\mu I-b R,
\end{aligned}
$$

onde S, I e R representam respectivamente as populações de suscetíveis, infectados e recuperados. Assume-se que indivíduos recuperados estão imunes, e os infectados são automaticamente infectantes.

\footnotetext{
${ }^{1}$ DeFEM, UNIJUÍ. Ijuí (RS). e-mail : emmendorfer@lycos.com

${ }^{2}$ CCNE, UFSM. Santa Maria (RS). e-mail : luizdiaz@ccne.ufsm.br
} 
Existe um importante parâmetro que determina o número de casos secundários produzidos a partir de um indivíduo infectado. Chamamos este parâmetro de taxa de reprodução básica $\left(R_{0}\right)$. Neste modelo podemos expressá-lo da seguinte forma [4]:

$$
R_{0}=\frac{\beta}{b+\mu} .
$$

Tanto este quanto outros modelos clássicos não consideram a variável espacial no espalhamento da epidemia. Pelo contrário, é comum supor que as populações de suscetíveis e infectados se misturem homogeneamente. Quando for válida esta suposição, então o termo $\beta S I$ vai representar o contato entre indivíduos suscetíveis e infectantes. Esta relação é chamada de lei de ação das massas, e tem origem no estudo da cinética química.

A dinâmica espacial deve, entretanto, ser considerada em muitos casos. No caso de populações pequenas e dispersas ou com baixa mobilidade é razoável admitir que a mistura entre infectados e suscetíveis não seja homogênea. Além disso, para estudar o espalhamento geográfico de uma epidemia é obrigatório que haja no modelo alguma forma de representação espacial.

A questão principal é como representar esta dinâmica espacial. Muitos dos modelos existentes utilizam Equações Diferenciais Parciais [5]. Recentemente, porém, uma proposta diferente têm sido adotada, onde a população é representada como um autômato celular [3] . Nesta abordagem as variáveis de estado do sistema, assim como o tempo, são discretos. O sistema é representado espacialmente através de um reticulado de células que interagem obedecendo a algumas regras de mudança de estado. A dinâmica do sistema como um todo depende desta interação local entre células.

Estando representada a dinâmica espacial podemos pesquisar o modelo para responder a algumas questões de interesse biológico relacionadas ao espalhamento geográfico de uma epidemia.

\section{Descrição do Modelo}

Utilizamos um modelo autômato celular para estudar o avanço de epidemias. O intervalo $\Delta t$ representa o passo discreto de tempo adotado. Cada célula representa um indivíduo, que pode estar em um entre três estados : suscetível (S), infectado (I) e recuperado (R). A transmissão da doença é representado como sendo um processo probabilístico. Cada indivíduo infectado tem igual e independente probabilidade, $q$, de infectar um outro indivíduo com o qual tenha contato.

Ou seja, a chance de que um indivíduo suscetível se torne infectado vai depender do número de contatos que ele estabelece com outros indivíduos no intervalo de tempo $\Delta t$, e também da probabilidade q de que cada contato resulte em transmissão. O número de contatos por unidade de tempo é um parâmetro característico de modelos baseados em indivíduos, e vamos designá-lo por $N_{c}$. A probabilidade de que um indivíduo se recupere da doença deve ser também representada, assim como a probabilidade de morte. Portanto as probabilidades de transição de estado 
são:

$\begin{array}{lll}\text { transição } & \text { interpretação } & \text { probabilidade } \\ S \Rightarrow I & \text { contágio } & 1-(1-q) N_{c} \\ I \Rightarrow R & \text { recuperação } & \mu \\ I \Rightarrow S ; R \Rightarrow S & \text { morte } & b\end{array}$

A população é constante, por isso quando um indivíduo morre, consideramos que nasce um suscetível na mesma célula que ele ocupava. Além disso, a população é representada como sendo uniformemente distribuída no espaço.

Um modelo semelhante foi proposto por Holmes [3]. Em seu modelo, dois padrões gerais de contato poderiam ser representados. No primeiro padrão os contatos são estritamente locais. Ou seja, um indivíduo só pode manter contato e transmitir a doença para seus vizinhos mais próximos no reticulado. O outro padrão de contato representa uma população que se mistura homogeneamente, onde se pode admitir que todos os indivíduos estão em contato entre si. Para este último caso, Holmes mostra que o autômato celular se comporta de maneira muito parecida com o modelo SIR baseado em equações diferenciais de Kermak-McKendrick (1.1). Por outro lado, quando apenas os contatos locais são considerados, o resultado é diferente em vários aspectos. Ambos padrões de contato representam situações extremas onde ou há pouca movimentação de indivíduos, ou esta movimentação é intensa.

Para preencher a lacuna que existe entre estes dois casos extremos, incluímos neste modelo parâmetros que permitem representar contatos não-locais. Designaremos por $p_{n l}$ a probabilidade de que um contato não local seja feito durante o tempo $\Delta t$. Vamos admitir que este contato seja feito com algum indivíduo que esteja a uma distância $L$ no reticulado (figura 1 ). O valor de $L$ deverá estar relacionado com a distância média que um indivíduo percorre durante uma movimentação, enquanto que a probabilidade $p_{n l}$ está relacionada a chance de que esta movimentação ocorra. Sabemos que esta probabilidade vai depender de muitos fatores, inclusive sazonalidade. Neste modelo, porém, iremos considerar $p_{n l}$ como sendo constante.

Com isso, número médio de contatos que um indivíduo faz em uma iteração é:

$$
N_{c}=N_{l o c}+p_{n l},
$$

onde $N_{l o c}$ é o número de contatos estritamente locais.

Para o modelo original de Holmes [3] a taxa reprodutiva básica de uma doença é dada por :

$$
R_{0}=\frac{q N_{l o c}}{\mu+b}
$$

ou seja, é proporcional ao número de contatos feitos. No nosso modelo, o número de contatos é dado por (2.1), logo a taxa reprodutiva básica é :

$$
R_{0}=\frac{q N_{c}}{\mu+b} .
$$

Fenômenos tais como espalhamento de epidemias podem ser representados de modo similar a invasões biológicas $[1,2]$. Desta forma o padrão de contato não local 


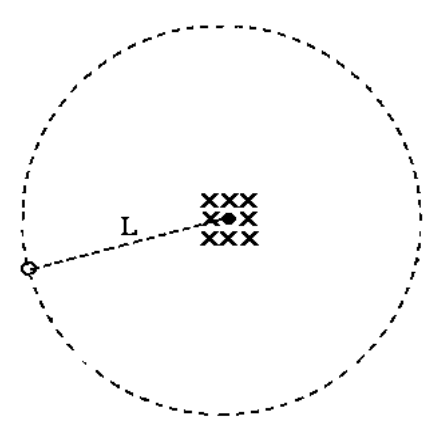

Figura 1: Padrões de contato. O indivíduo indicado com uma bola cheia está em permanente contato com seus oito vizinhos mais próximos (assinalados com $x$ ). Entretanto existe uma probabilidade $p_{n l}$ de que, a cada iteração, este indivíduo faça um contato não local (bola vazia). Este contato será feito com algum indivíduo na circunferência de raio $L$. Nas simulações, sempre que há um contato não local é feito um sorteio entre todos as possíveis células na circunferência, para que se determine qual delas será alvo do contato. Como o espaço é discretizado, pode acontecer que a distância $L$ seja tomada aproximadamente.

que foi incluído no modelo através dos parâmetros $L$ e $p_{l n}$ pode ser comparado a uma forma de colonização com migrantes de longa distância, tal como acontece no artigo de Shigesada [6] a respeito de invasões biológicas. Neste modelo, a colônia inicial da espécie invasora cresce a uma velocidade constante, ao mesmo tempo que gera novas colônias. O processo de colonização inclui a gradativa fusão da colônia mãe com as colônias filhas à medida que ambas crescem.

\section{Resultados}

Nas simulações realizadas procuramos observar o avanço de um pequeno foco de uma doença sobre uma população de suscetíveis. Iniciamos as simulações com apenas um infectado no centro do reticulado, e os demais são suscetíveis. Para variar $R_{0}$ percebemos, através da equação (2.3), que algum dos parâmetros $q, b$, ou $\mu$ deveria ser escolhido para que seu valor fosse dado em função de $R_{0}$. O parâmetro escolhido então foi $q$.

Já quando variamos o valor de $L$, usamos sempre uma probabilidadade pequena de contato não-local $\left(p_{n l}=0,1\right)$. Quando dizemos que $L=0$, deve-se assumir que também $p_{n l}=0$. Mesmo com essa probabilidade pequena, é possível notar que os contatos não-locais exercem um papel fundamental no espalhamento da epidemia.

A evolução dos contingentes populacionais ao longo do tempo está representada na figura 2. Notamos que o parâmetro $L$ exerce forte influência sobre a periodicidade da epidemia.

Na figura 3 apresentamos padrões espaciais de espalhamento da epidemia. Nos dois gráficos onde $L \neq 0$ ocorre a formação de focos secundários. À medida que estes 

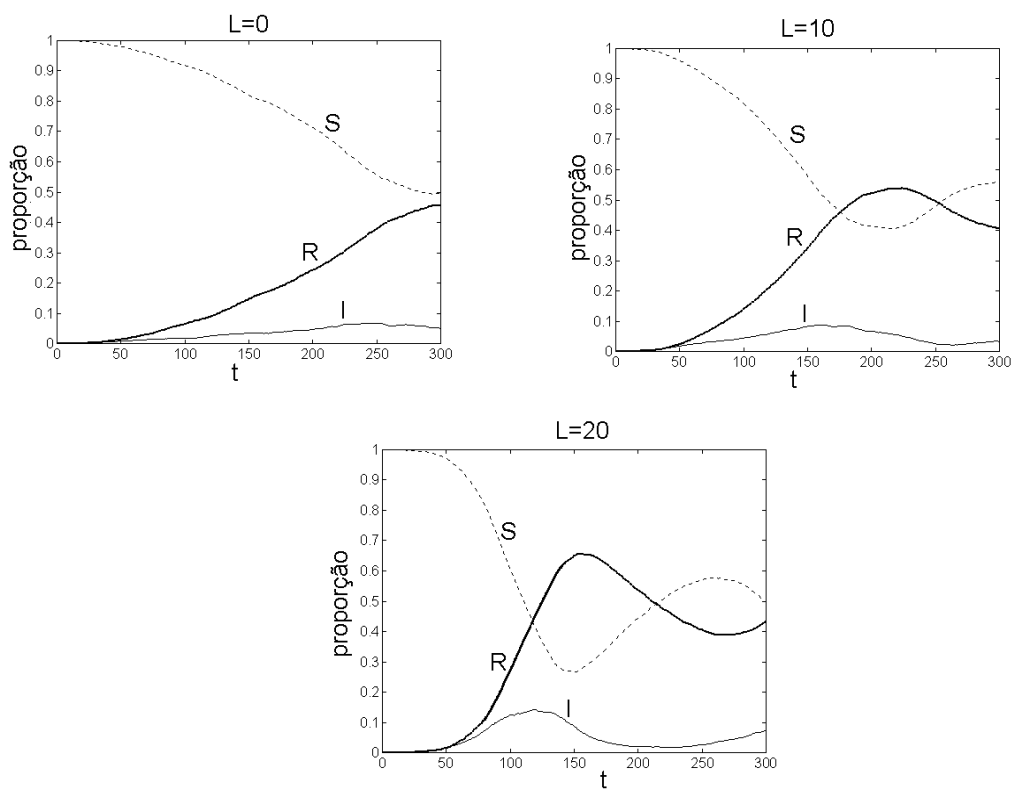

Figura 2: Evolução dos contingentes populacionais ao longo do tempo para diferentes valores de $L$. Em todas as figuras $t=100$. Para todas as simulações os parâmetros são os seguintes : $R_{0}=5 ; b=0,01 ; \mu=0,1 ; N_{l o c}=8$; A linha pontilhada representa a proporção de suscetíveis $(\mathrm{S})$; a linha mais grossa indica a proporção de recuperados $(\mathrm{R})$ e a linha mais fina representa a proporção de infectados(I). As proproções são calculadas em relação à população total.

focos crescem, eles vão fundindo-se com a colônia principal. Este comportamento é observado também por Shigesada [6] em seu modelo sobre invasões biológicas.

Os gráficos da figura 4 mostram um comportamento médio apresentado pelo modelo. Ou seja, o eixo vertical representa a quantidade média de infectados em função da distância do indivíduo à origem, sendo esta média calculada depois de 30 simulações. Este comportamento médio fornece resultados que nos permitem observar um caráter determinístico que surge a partir de um modelo que é probabilístico.

Os perfis de onda mostrados na figura 4 parecem sugerir que a tendência natural do modelo corresponde a uma solução do tipo onda viajante. Todos os perfis mostrados levam um certo tempo adquirir sua forma definitiva, após o quê permanecem aproximadamente sem modificação, transladando-se em oposição à origem. Basta apenas verificar se a velocidade com que as ondas se transladam é constante.

Esta verificação poderia ser feita com o auxílio da figura 5, que mostra a posição da onda em função do tempo. No trecho final das curvas a evolução parece linear, porém não podemos afirmar com certeza.

Murray [5] apresenta um modelo para o espalhamento de epidemias com equações 


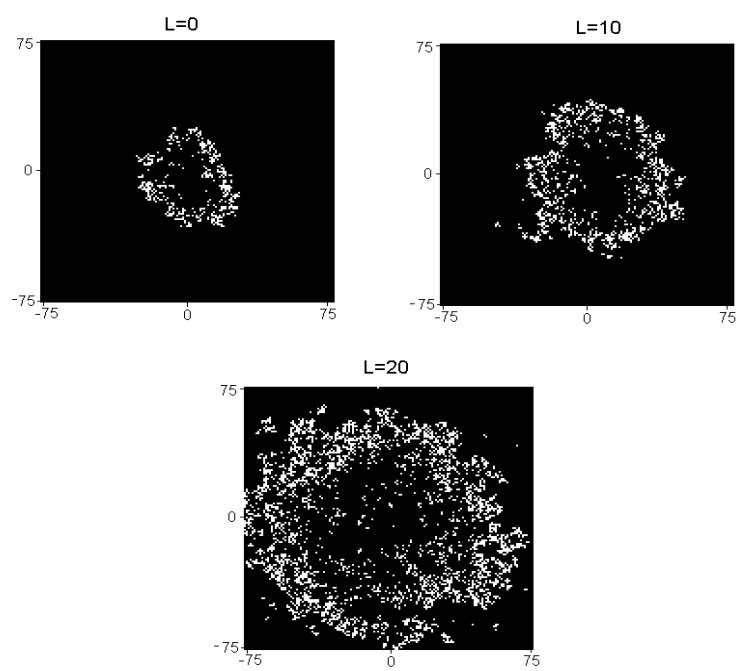

Figura 3: Exemplos de padrões espaciais obtidos. As simulações representadas são as mesmas que na figura 1 . Todos os gráficos representam o tempo $t=150$. Suscetíveis, Infectados e Recuperados são representados pelas cores preta, branca e cinza, respectivamente.
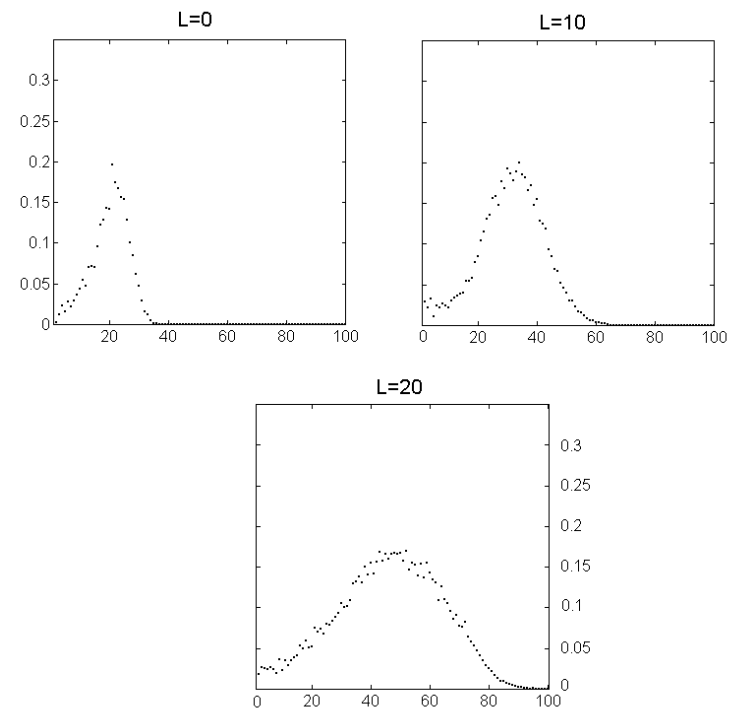

Figura 4: Perfis de onda para diferentes valores de $L$, com os mesmos parâmetros usados na figura 1 


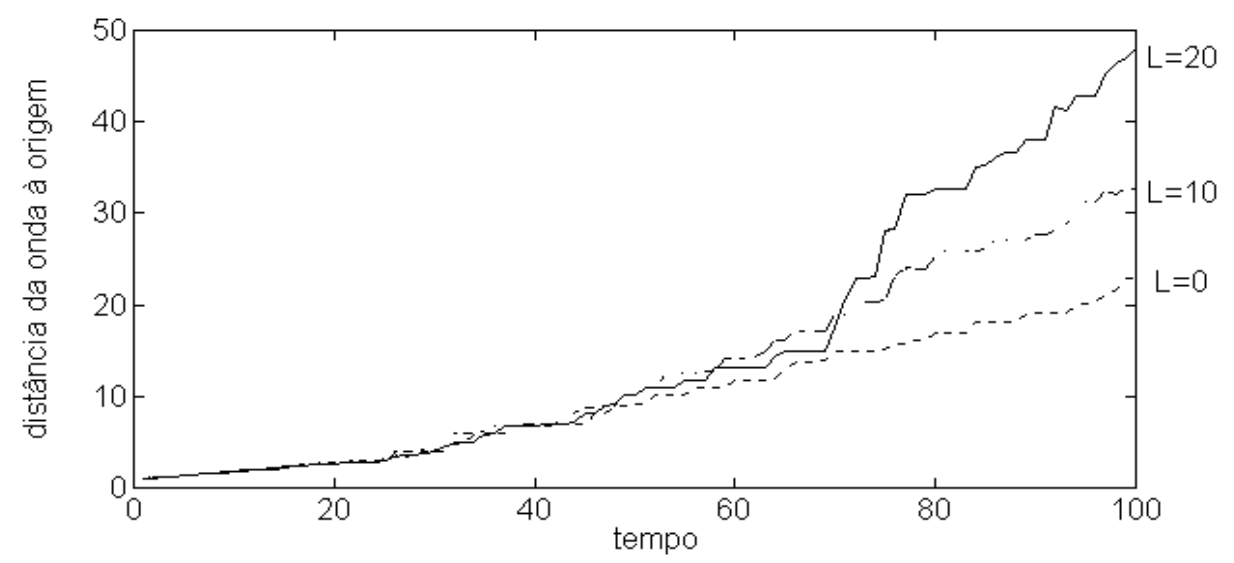

Figura 5: Distância das ondas em relação à origem em função do tempo.

diferenciais parciais. Em seu modelo a solução é do tipo ondas viajantes, com velocidade constante. Aparentemente o nosso modelo apresentou o mesmo comportamento.

A curva com $L=20$ mostrada na figura 5 ilustra o fato de as ondas demorarem a se formar. Ela cresce de modo semelhante às outras duas, estando inclusive inferior à curva de $L=10$, até que em aproximadamente $t=70$ ela dá um salto. Ocorre que em torno daquele instante de tempo a onda passa a englobar colônias próximas, que crescem isoladas da colônia original. Dali em diante aquela onda não muda muito com o tempo.

A velocidade aproximadamente constante $c$ das ondas viajantes foi calculada para alguns valores de $L$ :

\begin{tabular}{|c|c|c|c|c|c|c|}
\hline$L$ & 0 & 5 & 10 & 15 & 20 & 25 \\
\hline$c$ & 0,26 & 0,33 & 0,48 & 0,68 & 0,94 & 1,31 \\
\hline
\end{tabular}

O parâmetro $R_{0}$ também influi na velocidade da onda:

\begin{tabular}{|l|l|l|l|l|l|l|l|l|l|l|l|}
\hline$R_{0}$ & 3 & 3,2 & 3,4 & 3,6 & 3,8 & 4 & 4,2 & 4,4 & 4,6 & 4,8 & 5 \\
\hline$c$ & 0,10 & 0,12 & 0,14 & 0,15 & 0,16 & 0,16 & 0,17 & 0,18 & 0,20 & 0,22 & 0,24 \\
\hline
\end{tabular}

Abstract. In general, the classical Epidemiology theory does not take into account the spatial dimension of disease transmission, and instead it assumes that each individual has an equal probability of contacting the others. Spatial models are unavoidable when questions such as the speed at which a disease spreads and the spatial pattern of prevalence of that disease are analysed. Holmes [3] considers a cellular automata $(\mathrm{CA})$ model for the geographic spread of epidemics. In that model, the transmission is strictly local. The model is compared with a global transmission model. In our work, we study other ways to represent the disease 
transmission among individuals in the CA model. Besides the "local"neighborhood, witch includes the geometrically nearest individuals, we included "nonlocal" effects, representing the aleatory contacts that may occur among distant individuals. The relative variation between the local and global transmission scales may allow us to analyse several situations of interest in epidemic dynamics.

\section{Referências}

[1] O. Dickmann, Thresholds and travelling waves for the geographic spread of infection, J. Math. Biol. 6 (1978), 109-130.

[2] D.R. Hart, A spatial model for the spread of invading organisms subject to competition, J. Math. Biol. 35 (1997), 935-948 .

[3] E.E. Holmes, Basic epidemiological concepts in a spatial context, in "Spatial Ecology: the Role of Space in Population Dynamics and Interspecific Interactions" (D. Tilman and P. Kareiva, eds.), pp. 111-136, Princeton University Press, Princeton, 1997.

[4] W.O. Kermack e A.G. McKendrick, A contribution to the mathematical theory of epidemics, Proceedings of the Royal Society of London Series A 115 (1927), 700-721.

[5] J.D. Murray, "Mathematical Biology", Springer-Verlag, New York, 1989.

[6] N. Shigesada, Modeling stratified diffusion in biological invasion, The American Naturalist 146 (1995), 229-251. 